\title{
Tinmith-Hand: Unified User Interface Technology for Mobile Outdoor Augmented Reality and Indoor Virtual Reality
}

\author{
Wayne Piekarski and Bruce H. Thomas \\ Wearable Computer Laboratory \\ School of Computer and Information Science \\ University of South Australia \\ Mawson Lakes, SA, 5095, Australia \\ \{wayne,thomas\}@cs.unisa.edu.au
}

\begin{abstract}
This paper presents a unified user interface technology, using $3 D$ interaction techniques, constructive solid geometry, and a glove based menuing system, known as TinmithHand, to support mobile outdoor augmented reality applications and indoor virtual reality applications. TinmithHand uses similar concepts for both domains so that AR and VR applications are consistent and simple to use. The future goal of this user interface is to allow collaboration between outdoor AR and indoor VR systems.
\end{abstract}

Keywords: augmented reality, user interface, virtual reality

\section{Introduction}

We have been investigating immersive 3D outdoor Augmented Reality (AR) architectures and applications. One area we have investigated is the collaboration between a user outdoors operating an AR application, while a second user is indoors in a Virtual Reality (VR) setting [2]. We believe a unified user interface technology between outdoor $\mathrm{AR}$ and indoor VR applications is desirable to facilitate the interoperability between the systems.

Previously, we have demonstrated a mobile user of an AR system interacting with outdoor 3D entities, while another user interacts with the same entities on a VR workstation situated indoors [2]. In this demonstration, multiple systems are connected together via a wireless network, and share the same information. AR users are represented as avatars on the VR display, and virtual objects are overlaid as labels and points on the user's outdoor view. We provide the functionality of virtual cameras without the need to transmit large amounts of raw video data, with only position and orientation information being transmitted.

The goal of our current investigations is to extend our previous work and support collaboration between users in an indoor VR setting, and mobile users outdoors viewing the same information with an AR interface. Imagine someone is sent to a remote location to survey the physical terrain and building features; simultaneously experts are viewing the data feed and directing the remote person's actions. The remote person is recording the surveyed information via a mobile AR computing system. The indoor experts view the model as it is being built, and therefore the experts can direct the remote users to regions of most interest by annotating the remote user's view of the world. By using a single user interface to control both AR and VR environments, users can easily switch systems with reduced cognitive load and training time.

\subsection{Contribution}

In this paper we present one possible user interface, Tinmith-Hand, for the two users, which uses pinch gloves and thumb tracking to control a menu and a $3 \mathrm{D}$ modelling system. The annotation and communication aspects of the above scenario are currently being investigated. The modelling and recording aspect has been demonstrated as two applications using this user interface, Tinmith-Metro (an outdoor AR application [3]) and Tinmith-VR (an indoor VR application presented here and in [4]). Tinmith-Metro allows users to capture the designs of outdoor buildings and structures using beyond arms reach manipulation techniques. Tinmith-VR is designed to model virtual objects in the same fashion as Tinmith-Metro, but also allows for direct manipulation operations that are within arms reach. Both modellers are based around new techniques we have developed which rely on the intuitive nature of existing constructive solid geometry (CSG) operations, such as the infinite planes technique demonstrated in [3]. These capturing techniques are designed to create simple representations of objects faster than conventional surveying methods.

The contribution of this work is the single user interface technology to support interactive CSG modelling, as well as the way CSG is applied to capture outdoor $3 \mathrm{D}$ structures with AR, and create new models indoors with VR. Our work draws heavily on known good solutions to AR and VR interaction problems, and it is through the combination of these solutions that our technology has been developed. These are described further in [3].

\section{Interactions}

Our main interactions with Tinmith-Hand are through head and hand gestures, since we wish to keep the hands 
free from holding input devices if possible, allowing more natural styles of interaction. To control the user interface, the user's head and hands are tracked in 3D space, and finger pinches are used to control a menuing system. This menu system is described in [3], and was designed to handle more complex applications than comparable menu systems such as [1]. A menu is fixed to the bottom of the display, and each finger maps to one of eight dynamic menu items, controlling the entire system.

When the menu system is combined with $3 \mathrm{D}$ cursors located on the user's hands and their line of sight, it is possible to interact with 3D environments in an efficient and natural manner. We defined an eye cursor for specifying objects and planes along the line of sight relative to the body. The one handed cursor is useful for both selection and translation, while the two handed cursor is used for multiple selections and relative rotations and scaling.

Our system supports both image plane techniques [5] (for beyond arms reach) and also direct manipulation of objects (within arms reach). These techniques are used for both the AR and VR cases, allowing selection and transformation of existing $3 \mathrm{D}$ objects using natural hand motions. The primary interaction in the outdoor AR case is based on image plane techniques, while the VR case is primarily based on direct manipulation. For changing camera locations, users physically walk to the required location in the AR situation, whereas in VR the user travels to this location using flying or some other method, through a world that has been scaled for viewing.

It is possible to create new objects - either prefabricated models or those built by the user with simple primitives. The infinite planes technique allows users to create complex shapes like buildings using only infinite planes placed at appropriate locations. When combined with CSG operations such as carving, it is possible to capture many common shapes. We believe these operations are intuitive because they are based on real world actions.

\section{Tinmith-VR indoor modelling example}

Figure 1 shows a screen capture of Tinmith-VR being used to create a sample scene. In this example, the user is creating a scale model of a small building, such as a barn on a farm. This building has an arbitrarily curved roof, and so it is not possible to use a cube or a prefabricated object.

To capture this building, the user's body and hands are tracked using a Polhemus 6DOF tracker. The user defines the curve of the roof by specifying a set of infinite planes with pinches of the non-dominant hand. Initially, these planes are separate entities, but when combined together using the CSG intersect operation, we can form a solid shape. The solid is defined by the convex region that is enclosed by all the planes. If more complex concave shapes are required, the object must be created in parts and merged together. The CSG object can be modified interactively, allowing the user to make final adjustments to the planes before saving the final object. To complete the model, the user adds some extra models such as tables, street lamps, and pine trees. These models were prefabricated in another application, but could also have been created using this system if desired.

\section{Conclusion}

This paper has introduced the Tinmith-Hand AR/VR unified user interface. Using our modelling techniques, based on CSG, tracked input gloves, image plane techniques, and a menu control system, it is possible to build applications that can be used to construct complex 3D models of objects in both indoor and outdoor settings, sharing a common interface, and designed for collaborative applications.

We are currently investigating a search and rescue application, where outdoor users can mark in damage and changes to buildings. Remote experts can guide these mobile users to record specific information and improve situational awareness of the operations coordinators.

\section{References}

[1] Bowman, D. A. and Wingrave, C. A. Design and Evaluation of Menu Systems for Immersive Virtual Environments. In IEEE Virtual Reality 2001, pp 149-156, Yokohama, Japan, Mar 2001.

[2] Piekarski, W., Gunther, B., and Thomas, B. Integrating Virtual and Augmented Realities in an Outdoor Application. In 2nd Int'l Workshop on Augmented Reality, pp 45-54, San Francisco, Ca, Oct 1999.

[3] Piekarski, W. and Thomas, B. Tinmith-Metro: New Outdoor Techniques for Creating City Models with an Augmented Reality Wearable Computer. In 5th Int'l Symposium on Wearable Computers, Zurich, Switzerland, Oct 2001.

[4] Piekarski, W. and Thomas, B. Unifying Augmented Reality and Virtual Reality User Interfaces. Technical Report, University of South Australia, Mawson Lakes, SA, Jan 2002.

[5] Pierce, J., Forsberg, A., Conway, M., Hong, S., Zeleznik, R., and Mine, M. Image Plane Interaction Techniques in 3D Immersive Environments. In 1997 Symposium on Interactive 3D Graphics, pp 39-43, Providence, RI, Apr 1997.

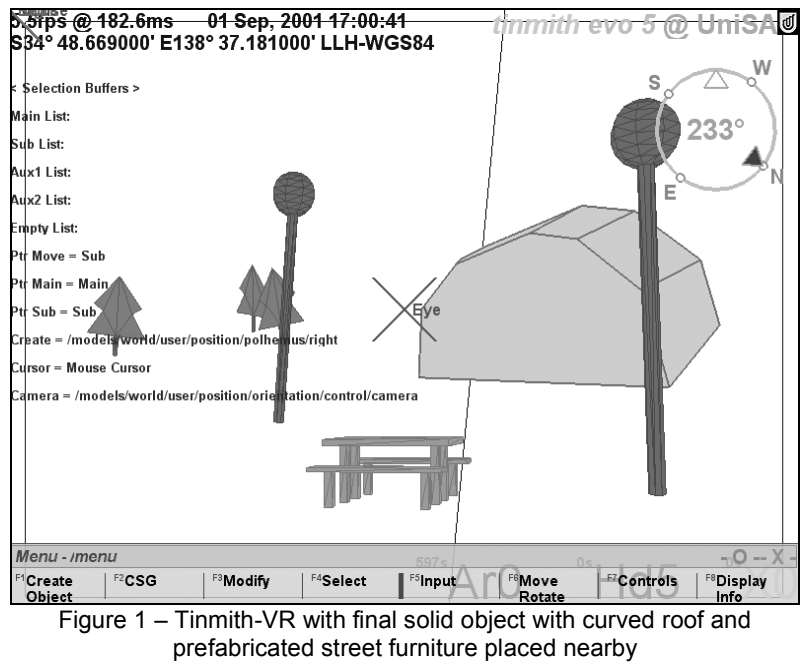

